\title{
A 6 to 10 Years Follow-Up Study Comparing Two-incision Total Hip Arthroplasties in Patients with Osteonecrosis of the Femoral Head or Hip Dysplasia
}

\author{
Hou Tsung Chen, MD ${ }^{1, *} \quad$ Meng-Lin Lu, MD ${ }^{1, *}$ Feng-Chih Kou, MD ${ }^{1} \quad$ I-Der Lu, MD ${ }^{1}$ Po-Chun Lin, MD ${ }^{1}$ \\ Mel S. Lee, MD, $\mathrm{PhD}^{1}$ \\ ${ }^{1}$ Department of Orthopaedic Surgery, Kaohsiung Chang Gung \\ Memorial Hospital, Kaohsiung City, Taiwan \\ J Hip Surg 2017;1:33-37.

\begin{abstract}
Address for correspondence Mel S. Lee, MD, PhD, Department of Orthopaedic Surgery, Kaohsiung Chang Gung Memorial Hospital, 123, Ta-Pei Rd., Niao-Sung, Kaohsiung, Taiwan

(e-mail: bone@doctor.com; mellee@cgmh.org.tw).
\end{abstract}

\begin{abstract}
Two-incision total hip arthroplasty (THA) has a steep learning curve and might increase the risks of implant malposition and perioperative complications in difficult cases. Whether dysplasic hip or osteonecrosis of the femoral head (ONFH) would have different results by the two-incision technique remains unreported. From 2003 to 2010, 159 hips in 151 patients (68 female and 83 male) treated with the two-incision THA were included. Cases were divided into two groups with ONFH in 99 hips and dysplasia in 60 hips. The inclusion criteria for hip dysplasia were a Sharp's angle more than $43^{\circ}$ and coverage of the femoral head less than $75 \%$. Clinical data and radiological measurement were retrospectively analyzed. The ONFH patients had younger age (48.5 \pm 12.8 years) with male predominance $(74 \%)$ while the dysplasic patients had older age (62 \pm 12 years) with female predominance (76\%). There were no differences in preoperative functional score, operation time, blood loss, wound size, and length of hospital stay between groups. The ONFH group had bigger cup size $(p=0.028)$ but similar stem size $(p=0.072)$ as compared with the dysplasia group. The cup inclination angle was $43.7^{\circ} \pm 4.8^{\circ}$ and $42.8^{\circ} \pm 5^{\circ}(p=0.25)$ and the cup anteversion angle was $17.6^{\circ} \pm 7.6^{\circ}$ and $14.2^{\circ} \pm 8.2^{\circ}(p=0.009)$, in the ONFH and the dysplasia group, respectively. At the final follow-up, there were three revisions cases (one septic

\section{Keywords}

- two-incision technique

- dysplasia

- total hip arthroplasty

- osteonecrosis loosening in the dysplasia group and two periprosthetic fractures in the ONFH group). No dislocation was noted in the study cohort. We had overall 99.4\% cup and $98.1 \%$ stem survival rate with the two-incision THA. The only differences were the bigger cup size and the more anteverted cup position in the ONFH hips as compared with the dysplasic hips. The two-incision THA seemed to be successful for patients with ONFH or dysplasic hips.
\end{abstract}

Muscle sparing approaches for total hip arthroplasties (THAs) have been advocated in the past two decades. The twoincision approach technique is one of the muscle sparing approaches. The technique has the potential benefits of avoiding muscle and tendon damage, less intraoperative blood loss, rapid patient recovery, and shortened hospitali-

HTC and MLL contributed equally to this paper as the first authors. zation but might increase complications such as fractures, nerve injury, and implants malposition, so it required a steep learning curve. ${ }^{1-6}$ The ideal cup position, stem alignment, and proper leg length and offset are the keys to a successful THA. Our previous study has demonstrated that using of intraoperative fluoroscopy or imageless navigation system can increase the accuracy of cup and stem alignment for the two-incision THA. ${ }^{7}$
Guest Editors: Lynne C. Jones, PhD and Michael A. Mont, MD
Copyright $\odot 2017$ by Thieme Medical Publishers, Inc., 333 Seventh Avenue, New York, NY 10001, USA. Tel: +1(212) 584-4662.
DOI http://dx.doi.org/ 10.1055/s-0036-1597963. ISSN 2472-8446. 
Osteonecrosis of the femoral head (ONFH) and hip dysplasia often lead to end stage hip osteoarthritis and need THAs. Different to ONFH, hip dysplasia have altered acetabular version and femoral torsion. The altered anatomy may increase the difficulties of implant positioning especially with the muscle sparing two-incision technique that the surgical field exposure is restricted. In the medical literature, a study comparing the clinical outcomes of the two-incision THA between ONFH and hip dysplasia patients has not been reported. The purpose of this study was to investigate whether the clinical outcomes, the implant positions, and the complications would be different by using the two-incision THA on patients with ONFH or hip dysplasia.

\section{Material and Methods}

From September 2003 to March 2010, a single surgeon had performed 175 two-incision THAs on 157 patients. ${ }^{8}$ By excluding cases with primary osteoarthritis, ankylosing spondylitis, rheumatoid arthritis, Legg-Calve-Perthes disease, and posttraumatic arthritis, 159 hips in 151 patients (68 female and 83 male) were included as the study cohort and were divided into 2 groups. Group 1 had 99 hips (93 patients) with ONFH. Group 2 had 60 hips (58 patients) with hip dysplasia. Hip dysplasia was defined based on the criteria of Sharp's angle more than $43^{\circ}$ and the coverage of the femoral head less than $75 \%{ }^{9}$ Only mild dysplasic hips in the Crowe I or II classes were included. ${ }^{10}$ Demographic data including age, gender, body mass index, preoperative Harris hip score (HHS) and the Western Ontario and McMaster University Osteoarthritis Index (WOMAC) ${ }^{11}$ were recorded ( - Table $\mathbf{1}$ ). All THAs were performed with the two-incision technique as described previously. ${ }^{7}$ The operative time, amount of blood loss, wound length, length of hospital stay, size of implants, and complications were recorded. Postoperatively, patients were encouraged to ambulate as soon as possible by protected weight bearing with double crutches for 6 weeks and a single crutch for another 6 weeks. Clinical follow-up included radiological examinations, HHS, and WOMAC scale at 3 weeks, 6 weeks, 12 weeks, 6 months, and yearly after the surgery.

Radiological analysis included standard pelvis anteroposterior radiograph taken postoperatively and at 3 months, 6 months, and yearly in the follow-up. The angle of cup inclination and anteversion, the stem alignment, and the ratio of canal filling were recorded according to the standard protocols. ${ }^{12-14}$ Any implant migration, loosening, or early failure of the components were recorded.

\section{Results}

All 159 hips were followed with functional and radiographic studies. The age and gender distribution were significantly different with younger age ( $48.5 \pm 12.8$ years) and predominantly male gender $(74 \%)$ in the ONFH group as compared with older age (62 \pm 12 years) and predominantly female gender (76\%) in the dysplasia group (-Table 1 ). The preoperative HHS and WOMAC scale were similar in both groups.

There were no differences in regard to the operation time, blood loss, wound size, length of hospital stay, and the postoperative HHS or WOMAC scale between the two groups (-Table 2). The ONFH group had more male patients and the cup size was significantly bigger $(p=0.028)$ with a trend of larger stem size $(p=0.072)$ as compared with the dysplasia group. The cup inclination angle was $43.7^{\circ} \pm 4.8^{\circ}$ in the $\mathrm{ONFH}$ group and $42.8^{\circ} \pm 5^{\circ}$ in the dysplasia group $(p=0.25)$ and the cup anteversion angle was $17.6^{\circ} \pm 7.6^{\circ}$ in the ONFH group and $14.2^{\circ} \pm 8.2^{\circ}$ in the dysplasia group $(p=0.009)$. There was no difference in femoral canal fill ratio between the two groups (ONFH:94 $\pm 4 \%$, Dysplasia: $95 \pm 5 \% ; p=0.088$ ). With a mean follow-up of $9.6 \pm 1.5$ years, there were three revision cases in the cohort with one septic loosening in the dysplasia group and two stem revision in the ONFH group due to periprosthetic fractures. No dislocation in either group was noted in the study. The overall implant survival rate was $98 \%$ in the ONFH group and $98.3 \%$ in the dysplasia group, respectively. There were four patients with intraoperative fracture (two in greater trochanter and two in proximal femur) in the ONFH group. For the dysplasia group, there were six patients with intraoperative fracture (two in greater trochanter and four in proximal femur). The lateral femoral cutaneous nerve (LFCN) injury was noted in 26 cases in the ONFH group and eight cases in the dysplasia group. The incidence of LFCN injury was approximately $21 \%$ by using the two-incision THA technique.

\section{Discussion}

Muscle sparing approaches have become popular recently in the fields of joint arthroplasty and other orthopedic surgeries.

Table 1 Patient demographics

\begin{tabular}{|l|l|l|l|}
\hline & Group 1 (ONFH) & Group 2 (Dysplasia) & p-value \\
\hline Male: Female & $75: 24$ & $14: 46$ & \\
\hline Age (years) & $48.5 \pm 12.8$ & $61.5 \pm 12$ & $<0.001^{*}$ \\
\hline BMI & $24.2 \pm 3.5$ & $25.2 \pm 4.3$ & 0.112 \\
\hline \multicolumn{4}{|l|}{} \\
\hline Preoperative function \\
\hline Warris hip score & $56.8 \pm 12.0$ & $59.8 \pm 10.7$ & 0.090 \\
\hline
\end{tabular}

Abbreviation: BMI, body mass index = body weight/(body height)2

*Statistically significant difference at $p \leq 0.05$. 
Table 2 Surgical results and implant positions

\begin{tabular}{|l|l|l|l|}
\hline & Group 1 (ONFH) & Group 2 (Dysplasia) & p-value \\
\hline Operation time (min) & $157.6 \pm 45.2$ & $150.5 \pm 37.5$ & 0.303 \\
\hline Blood loss (mL) & $692.9 \pm 396.7$ & $617.7 \pm 219.0$ & 0.127 \\
\hline Wound length (cm) & $9.8 \pm 1.6$ & $9.8 \pm 1.6$ & 0.851 \\
\hline Hospital stay (days) & $5.6 \pm 2.2$ & $5.4 \pm 1.5$ & 0.499 \\
\hline Cup & \multicolumn{3}{|l|}{} \\
\hline Size $(\mathrm{mm})$ & $53.6 \pm 2.4$ & $52.7 \pm 2.8$ & $0.028^{*}$ \\
\hline Inclination ( $\left.{ }^{\circ}\right)$ & $43.7^{\circ} \pm 4.8^{\circ}$ & $42.8^{\circ} \pm 5^{\circ}$ & 0.254 \\
\hline Anteversion ( $\left.{ }^{\circ}\right)$ & $17.6^{\circ} \pm 7.6^{\circ}$ & $14.2^{\circ} \pm 8.2^{\circ}$ & $0.009^{*}$ \\
\hline Outlier & $5 / 99(5.1 \%)$ & $6 / 60(10 \%)$ & \\
\hline Stem & \multicolumn{3}{|l}{} \\
\hline Size(mm) & $12.6 \pm 1.5$ & $12.1 \pm 1.5$ & 0.072 \\
\hline Canal fill ratio (\%) & $95 \pm 5$ & 0.088 \\
\hline
\end{tabular}

Compared with conventional methods for THA, they theoretically use smaller wound, less soft tissue damage, shorter operative time, shorter hospital stays, less blood loss, and quicker recovery. ${ }^{15}$ Among the muscle sparing approaches, the two-incision approach had been enthusiastically advocated but gradually lost its popularity due to the increase risks of complications such as femoral fracture or implant malposition related to the limited visualization in the surgical fields and a steep learning curve. ${ }^{16,17}$ Fehring et al reported catastrophic complications by using this two-incision technique as a warning for inexperienced surgeons. ${ }^{16}$ Bal et al stated that the rates of complications such as femoral fracture, dislocation, lateral femoral cutaneous nerve injury, and repeat surgery associated with the two-incision technique for THA were very high even by a surgeon who was experienced with a single small incision for THA. ${ }^{18}$ Pagnano et al reported that the two-incision approach had higher complications (14\% versus $5 \%$ ) including calcar fractures, dislocation, and femoral nerve palsy as compared with posterior cases. ${ }^{19}$ As a contrast, Lee et al reported 2 to $3.1 \%$ intraoperative periprosthestic fracture rate for the twoincision THA and the complication rates could be diminished with increasing experience and use of intraoperative fluoroscopic guidance. ${ }^{7,20,21}$ There were 10 cases (6.3\%) with intraoperative femoral fractures, 34 cases $(21.4 \%)$ with temporary LFCN palsy, and 3 revision cases (one for septic loosening in the dysplasia group and two for the stem revision in the ONFH group for periprosthetic fracture). With a mean follow-up of 9 to 10 years, the overall implants survival rate is $98 \%$ in the ONFH group and $98.3 \%$ in the dysplasia group. We believed these results might be related to the modified technique we had used for the two-incision technique. First, we positioned our patients in the standard lateral decubitus position. The setting and orientation are more comfortable and familiar for surgeons who use direct lateral or posterior approach. Second, we changed the direction of the anterior incision wound $90^{\circ}$ to the original technique. Our incision therefore can span from the lesser trochanter to the greater trochanter that greatly improves the visualization of the surgical field for both the acetabulum and proximal femur. Yoon et al also reported excellent results by adopting a similar strategy in the patients positioning but used the Watson-Jones interval for the twoincision THAs. ${ }^{22}$

The original two-incision technique has the inherent difficulty of surgical field visualization, therefore an intraoperative fluoroscopy is routinely needed. It should be more cautious for the implant malposition especially in patients with hip dysplasia that underdeveloped acetabulum and excessive anteverted proximal femur are the common anatomic variations. Bal et al retrospectively compared two patient groups treated with two-incision THA or single-incision THA and found a substantially higher numbers of fractures, reoperation, cup malposition, and nerve injuries in the two-incision group. ${ }^{18}$ However, Amman et al found the position of acetabular component was more accurate in the MIS THA group because of the use of intraoperative imaging. ${ }^{23}$ Other researchers, including Williams et al, ${ }^{24}$ Teet et $\mathrm{al}^{25}$ and Siguier et $\mathrm{al}^{26}$ also claimed that mini-incision THA does not compromise the component position or dislocation. To our knowledge, no study reported the implant position, functional results, and complications in patients with relative normal anatomy (ONFH) or abnormal anatomy (hip dysplasia) by using the two-incision technique. This study analyzed 159 hips with ONFH or hip dysplasia and found the cup inclination, stem alignment; femoral canal fill ratio, hospital course, functional results, complications, and prosthesis survival were not different between them. The only differences were a relatively bigger cup in the ONFH patients (maybe gender related) and a less anteverted cup in the dysplasic hip patients. In the series, no major complications and no dislocation were found during the follow-up.

ONFH has been known to affect young male patients. ${ }^{27}$ On the other hand, developmental dysplasia of the hip is the common cause of secondary hip osteoarthritis and the prevalence ranges from 5.4 to $12.8 \%$ among different ethnic groups. ${ }^{28,29}$ The female gender is one of the known risk factors for dysplasic hips, and these patients usually end up 
with THA at younger age eventually. ${ }^{30}$ Due to undeveloped acetabulum, the acetabulum anatomy in dysplasic hip patients usually characterized with deficiencies in anterolateral and superior wall and lacks of bone stock medially. Because the ideal acetabular cup size and position are difficulty in dysplasic hip patients with THA, muscle sparing approaches such as the two-incision technique should not be recommended. In this study, only mild dysplasic hips in the Crowe I or II classes were included. As the final result, the average cup anteversion was $17.6^{\circ}$ in the ONFH group and $14.2^{\circ}$ in the dysplasic hip group. The majority of the cups were in the recommended safe zone with only $5.1 \%$ and $10 \%$ outliers, respectively. Nevertheless, no dislocation or cup loosening were found in this series. This may be due to the two-incision technique could preserve the tissue integrity and improve the tissue tension postoperatively. It was also interesting to note that the dysplasic hips had less anteverted cups as compared with the ONFH hips. The two-incision technique described herein used a modified incision that can effectively improve the surgical field exposure. By improving the exposure, we can visualize the trial components to determine the ideal compliant position by matching the cup and stem routinely..$^{31,32}$ The final position of the less anteverted cups in the dysplasic hips reflected the anatomic characteristics of anterolateral deficiency of acetabulum and more anteverted proximal femur. With these less anteverted and adequately inclined cups, the stability of the THAs in dysplasic hips could be well maintained.

The overall implant survival rate was satisfactory with $98.1 \%$ at $9.6 \pm 1.5$ years. We found by using the modified twoincision technique, patients with dysplasic hips could have similar results in the functional outcome, surgical results, and prosthesis survival as ONFH hips. However, there are many limitations of this study. The retrospective nature of this study could not conclude the merits of the two-incision technique to other approaches because case selection bias existed. Difficult patients with severe hip dysplasia, ONFH hips after salvage procedures, morbid obesity, or other comorbidities were not included in this study. The sample size was small with only 159 hips analyzed. The series was a single surgeon's experience that used a modified two-incision technique and could not represent a common scenario for the original two-incision technique. However, all cases could be followed up in the study period, and the results were analyzed independently. All cases used the same implants by the same technique. Most importantly, the modified twoincision technique with the improved surgical field exposure has not been critically analyzed on hips with different anatomic characteristics. In summary, we found the modified two-incision technique could achieve good to excellent clinical results and implant survival on selected patients with either ONFH or hip dysplasia

\section{References}

1 Berger RA. Total hip arthroplasty using the minimally invasive two-incision approach. Clin Orthop Relat Res 2003;(417):232-241
2 Van Oldenrijk J, Hoogervorst P, Schaap GR, van Dijk CN, Schafroth MU. Two-incision minimally invasive total hip arthroplastyresults and complications. Hip Int 2011;21(1):81-86

3 Zhang XL, Wang Q, Shen H, Jiang Y, Zeng BF. Minimally invasive two-incision total hip arthroplasty: a short-term retrospective report of 27 cases. Chin Med J (Engl) 2007; 120(13):1131-1135

4 Mardones R, Pagnano MW, Nemanich JP, Trousdale RT. The Frank Stinchfield Award: muscle damage after total hip arthroplasty done with the two-incision and mini-posterior techniques. Clin Orthop Relat Res 2005;441(441):63-67

5 Chou SW, Ueng SW, Lee MS. Muscular recovery of hip flexors and extensors after two-incision total hip arthroplasty. Chang Gung Med J 2008;31(6):576-582

6 Duwelius PJ, Burkhart RL, Hayhurst JO, Moller H, Butler JB. Comparison of the 2-incision and mini-incision posterior total hip arthroplasty technique: a retrospective match-pair controlled study. J Arthroplasty 2007;22(1):48-56

7 Lee MS, Kuo CH, Senan V, Chen WJ, Chen LH, Ueng SW. Twoincision total hip replacement: intra-operative fluoroscopy versus imageless navigation for cup placement. Hip Int 2006;16 (Suppl 4):35-41

8 Kellgren JH. Osteoarthrosis in patients and populations. BMJ 1961; 2(5243):1-6

9 Seol JH, Park KS, Yoon TR. Postoperative complications and costeffectiveness of simultaneous and staged bilateral total hip arthroplasty using a modified minimally invasive two-incision technique. Hip Pelvis 2015;27(2):77-82

10 Crowe JF, Mani VJ, Ranawat CS. Total hip replacement in congenital dislocation and dysplasia of the hip. J Bone Joint Surg Am 1979; 61(1):15-23

11 Harris WH. Traumatic arthritis of the hip after dislocation and acetabular fractures: treatment by mold arthroplasty. An endresult study using a new method of result evaluation. J Bone Joint Surg Am 1969;51(4):737-755

12 Zhao X, Lin T, Cai XZ, Yan SG. Comparison of minimally invasive surgery and mini-incision technique for total hip arthroplasty: a sub-group meta-analysis. Chin Med J (Engl) 2011;124(24): 4316-4323

13 Ogonda L, Wilson R, Archbold P, et al. A minimal-incision technique in total hip arthroplasty does not improve early postoperative outcomes. A prospective, randomized, controlled trial. J Bone Joint Surg Am 2005;87(4):701-710

14 Chan YS, Shih CH. Bipolar versus total hip arthroplasty for hip osteonecrosis in the same patient. Clin Orthop Relat Res 2000; (379):169-177

15 Berstock JR, Blom AW, Beswick AD. A systematic review and meta-analysis of the standard versus mini-incision posterior approach to total hip arthroplasty. J Arthroplasty 2014;29(10): 1970-1982

16 Fehring TK, Mason JB. Catastrophic complications of minimally invasive hip surgery. A series of three cases. J Bone Joint Surg Am 2005;87(4):711-714

17 Feinblatt JS, Berend KR, Lombardi AV Jr. Severe symptomatic heterotopic ossification and dislocation: a complication after two-incision minimally invasive total hip arthroplasty. J Arthroplasty 2005;20(6):802-806

18 Bal BS, Haltom D, AletoT, Barrett M. Early complications of primary total hip replacement performed with a two-incision minimally invasive technique. J Bone Joint Surg Am 2005;87(11):2432-2438

19 Pagnano MW, Leone J, Lewallen DG, Hanssen AD. Two-incision THA had modest outcomes and some substantial complications. Clin Orthop Relat Res 2005;441(441):86-90

20 Lee MS-S. Minimally invasive total hip arthroplasty. Formosan J Musculoskeletal Disord 2011;2(2):41-44

21 Lu ML, Chou SW, Yang WE, et al. Hospital course and early clinical outcomes of two-incision total hip arthroplasty. Chang Gung Med J 2007;30(6):513-520 
22 Yoon TR, Bae BH, Choi MS. A modified two-incision minimally invasive total hip arthroplasty: technique and short-term results. Hip Int 2006;16(Suppl 4):28-34

23 Amman S, Cizik A, Leopold SS, Manner PA. Two-incision minimally invasive vs standard total hip arthroplasty: comparison of component position and hospital costs. J Arthroplasty 2012;27(8):1569-1574.e1

24 Williams SL, Bachison C, Michelson JD, Manner PA. Component position in 2-incision minimally invasive total hip arthroplasty compared to standard total hip arthroplasty. J Arthroplasty 2008; 23(2):197-202

25 Teet JS, Skinner HB, Khoury L. The effect of the "mini" incision in total hip arthroplasty on component position. J Arthroplasty 2006; 21(4):503-507

26 Siguier T, Siguier M, Brumpt B. Mini-incision anterior approach does not increase dislocation rate: a study of 1037 total hip replacements. Clin Orthop Relat Res 2004;(426):164-173
27 Mont MA, Hungerford DS. Non-traumatic avascular necrosis of the femoral head. J Bone Joint Surg Am 1995;77(3):459-474

28 Kumar JN, Kumar JS, Wang VT, Das De S. Medium-term outcome of total hip replacement for dysplastic hips in Singapore. J Orthop Surg (Hong Kong) 2010;18(3):296-302

29 Papachristou G, Hatzigrigoris P, Panousis K, et al. Total hip arthroplasty for developmental hip dysplasia. Int Orthop 2006;30(1): 21-25

30 Weinstein SL, Mubarak SJ, Wenger DR. Developmental hip dysplasia and dislocation: Part II. Instr Course Lect 2004; 53:531-542

31 Dorr LD, Malik A, Dastane M, Wan Z. Combined anteversion technique for total hip arthroplasty. Clin Orthop Relat Res 2009; 467(1):119-127

32 Malik A, Maheshwari A, Dorr LD. Impingement with total hip replacement. J Bone Joint Surg Am 2007;89(8):1832-1842 